\title{
Article \\ Dysregulation of DNA Methylation and Epigenetic Clocks in Prostate Cancer among Puerto Rican Men
}

\author{
Anders Berglund ${ }^{1,+} \mathbb{D}$, Jaime Matta ${ }^{2,+} \mathbb{D}$, Jarline Encarnación-Medina ${ }^{2} \mathbb{D}$, Carmen Ortiz-Sanchéz ${ }^{2} \mathbb{D}$, Julie Dutil ${ }^{2}$ \\ Raymond Linares $^{2}$, Joshua Marcial ${ }^{2}$, Caren Abreu-Takemura ${ }^{2}$, Natasha Moreno ${ }^{2, *}$, Ryan Putney ${ }^{1}$, \\ Ratna Chakrabarti ${ }^{3} \mathbb{D}$, Hui-Yi Lin ${ }^{4}$, Kosj Yamoah ${ }^{5}$, Carlos Diaz Osterman ${ }^{2}$, Liang Wang ${ }^{6} \mathbb{D}$, Jasreman Dhillon ${ }^{7}$, \\ Youngchul Kim ${ }^{1}$, Seung Joon Kim ${ }^{8}$, Gilberto Ruiz-Deya ${ }^{2, \ddagger}$ and Jong Y. Park ${ }^{9, *, \ddagger \mathbb{D}}$
}

1 Department of Biostatistics and Bioinformatics, H. Lee Moffitt Cancer Center, Tampa, FL 33612, USA; anders.berglund@moffitt.org (A.B.); ryan.putney@moffitt.org (R.P.); youngchul.kim@moffitt.org (Y.K.)

2 Department of Basic Sciences, Ponce Research Institute, School of Medicine, Ponce Health Sciences University, Ponce 00716-2347, Puerto Rico; jmatta@psm.edu (J.M.); jencarnacion@psm.edu (J.E.-M.); carmenortiz@psm.edu (C.O.-S.); jdutil@psm.edu (J.D.); rlinares20@stu.psm.edu (R.L.); jmarcial20@stu.psm.edu (J.M.); cabreu20@stu.psm.edu (C.A.-T.); cjdiaz@psm.edu (C.D.O.); gruiz@psm.edu (G.R.-D.)

3 Burnett School of Biomedical Sciences, University of Central Florida, Orlando, FL 32816, USA; Ratna.Chakrabarti@ucf.edu

4 Biostatistics Program, School of Public Health, Louisiana State University Health Sciences Center, New Orleans, LA 70112, USA; hlin1@lsuhsc.edu

5 Department of Radiation Oncology, H. Lee Moffitt Cancer Center, Tampa, FL 33612, USA; Kosj.Yamoah@moffitt.org

Citation: Berglund, A.; Matta, J.;

Encarnación-Medina, J.;

Ortiz-Sanchéz, C.; Dutil, J.;

Linares, R.; Marcial, J.;

Abreu-Takemura, C.; Moreno, N.;

Putney, R.; et al. Dysregulation of DNA Methylation and Epigenetic

Clocks in Prostate Cancer among

Puerto Rican Men. Biomolecules 2022,

12, 2. https://doi.org/10.3390/

biom12010002

Academic Editor: Prakash Kulkarni

Received: 26 October 2021

Accepted: 14 December 2021

Published: 21 December 2021

Publisher's Note: MDPI stays neutral with regard to jurisdictional claims in published maps and institutional affiliations.

Copyright: (C) 2021 by the authors. Licensee MDPI, Basel, Switzerland. This article is an open access article distributed under the terms and conditions of the Creative Commons Attribution (CC BY) license (https:// creativecommons.org/licenses/by/ $4.0 /)$.
6 Department of Molecular Biology, H. Lee Moffitt Cancer Center, Tampa, FL 33612, USA; Liang.wang@moffitt.org

7 Department of Pathology, H. Lee Moffitt Cancer Center, Tampa, FL 33612, USA; Jasreman.Dhillon@moffitt.org

8 Department of Internal Medicine, Catholic University of Korea, Seoul 06591, Korea; cmcksj@catholic.ac.kr

9 Department of Cancer Epidemiology, H. Lee Moffitt Cancer Center, Tampa, FL 33612, USA

* Correspondence: advancedlaparascopy@gmail.com (N.M.); jong.park@moffitt.org (J.Y.P.)

$+\quad$ These authors equally contributed to this work as co-first author.

$\ddagger \quad$ These authors equally contributed to this work as co-last author.

Abstract: In 2021, approximately 248,530 new prostate cancer (PCa) cases are estimated in the United States. Hispanic/Latinos (H/L) are the second largest racial/ethnic group in the US. The objective of this study was to assess DNA methylation patterns between aggressive and indolent PCa along with ancestry proportions in $49 \mathrm{H} / \mathrm{L}$ men from Puerto Rico (PR). Prostate tumors were classified as aggressive $(n=17)$ and indolent $(n=32)$ based on the Gleason score. Genomic DNA samples were extracted by macro-dissection. DNA methylation patterns were assessed using the Illumina EPIC DNA methylation platform. We used ADMIXTURE to estimate global ancestry proportions. We identified 892 differentially methylated genes in prostate tumor tissues as compared with normal tissues. Based on an epigenetic clock model, we observed that the total number of stem cell divisions (TNSC) and stem cell division rate (SCDR) were significantly higher in tumor than adjacent normal tissues. Regarding PCa aggressiveness, 141 differentially methylated genes were identified. Ancestry proportions of PR men were estimated as African, European, and Indigenous American; these were $24.1 \%, 64.2 \%$, and $11.7 \%$, respectively. The identification of DNA methylation profiles associated with risk and aggressiveness of PCa in PR H/L men will shed light on potential mechanisms contributing to PCa disparities in PR population.

Keywords: prostate cancer; DNA methylation; aggressiveness; Hispanic/Latino population; ancestry structure

\section{Introduction}

Based on American Cancer Society data, 248,530 new prostate cancer (PCa) cases and 34,130 PCa-specific deaths are anticipated in the US in 2021 [1]. The lifetime risk of PCa is 
12.5\% [2]. PCa-specific mortality (PCSM) rates have been found to vary among different ethnic groups in the US. The study by Chinea et al. (2017) reported differences in PCSM rates when comparing Hispanic/Latino $(\mathrm{H} / \mathrm{L})$ subgroups to non-Hispanic Whites (NHWs) and non-Hispanic Blacks (NHBs) [3]. However, this study aggregated all H/L subgroups into one broad group including: Mexican Americans, Puerto Ricans, Cubans, South or Central Americans, and Dominicans. Therefore, we need to investigate variations in $\mathrm{H} / \mathrm{L}$ subgroup specific PCSM rates. Among different H/L subgroups, Puerto Rican (PR) men showed much higher PCSM rates than other Hispanic groups and NHBs [3]. Indeed, in Puerto Rico (PR), PCa is the most common type of cancer case and accounts for the most cancer-specific deaths [4].

$\mathrm{PCa}$ is a complex disease that is mediated by the accumulation of genetic and epigenetic aberrations, such as altered androgen receptor activity, changes in chromatin structure, differential expression of oncogenes and tumor suppressor genes, or defective cell division [5]. Differential DNA methylation can influence carcinogenesis and disease progression [6]. Indeed, the most common molecular event in PCa is dysregulation of DNA methylation. Among these epigenetic changes, some specific changes may be associated with poor outcomes, including PCSM, metastasis, and recurrence [7]. A study from the Cancer Genome Atlas (TCGA) found associations between gene expression and methylation profiles. This study suggested that epigenetic changes define distinct molecular subtypes of PCa [8]. The role of DNA methylation in promoter regions has been investigated many times, and most hypermethylation has been related with gene silencing of tumor suppressor genes in PCa and with poor outcomes [6,9-11]. PCa is typically known as a slowly developing disease. However, approximately 20\% of cases are classified as aggressive. The aggressive PCa phenotype is associated with the development of metastasis and poor survival outcomes.

Mateo et al. (2015) reported that the dysregulation of tumor suppressor genes are often found in aggressive PCa [5]. Promoter hypermethylation may drive cancer through tumor suppressor gene inactivation and activation of oncogenes [12]. Therefore, additional studies are needed to further understand the epigenetic regulation of tumor suppressor genes and oncogenes in PCa.

Numerous studies reported that differential DNA methylation influences the likelihood of developing PCa and also affects its progression [13,14]. Yang and Park (2012) conducted an extensive review of over 100 studies and presented a list of frequently reported differentially methylated genes in malignant prostate tissue [14]. Most of the studies reviewed investigated a small number of genes because of a small sample size in order to increase the chance to yield statistically significant results. However, this approach may not assess methylation impact associated with multiple genes. Several of the studies used an epigenome-wide methylation approach, often used to examine multiple genes. As expected, numerous differential methylated genes were identified. However, another data set is needed to validate findings [15-17]. Since differential DNA methylation may influence health disparities in PCa [18], there is a need to investigate methylation profiles to evaluate potential PR-specific methylated genes.

Aging is one of the major risk factors for PCa. The risk of carcinogenesis in any given tissue is closely related with the mitotic age of the tissue and therefore the cumulative number of cell divisions [19]. The turnover rate of tissues is affected by several factors, such as inflammation, injury, and exposure to carcinogens [20]. Therefore, increased turnover in tissues may increase molecular alterations and eventually lead to carcinogenesis [21]. DNA methylation may be involved in aging [22]. DNA methylation biomarkers for aging, also known as the epigenetic clock, have been developed based on DNA methylation data. DNA methylation age (DNAmAge), generated from the epigenetic clock, estimates epigenetic age, measures subject age, and, more importantly, predicts disease risk [23]. This DNAmAge can be used for predicting chronic diseases, including cancers, if these values show substantial deviations [24]. With this model, we can estimate epigenetic age and predict cancer risk [25]. In this study, the total number of stem cell divisions (TNSC) and 
stem cell division rate (SCDR) were compared between tumor and adjacent normal tissues using this method [25].

Currently, the dysregulation of DNA methylation in PR PCa patients is well known. We recently reported differentially methylated genes in PCa tumor tissues and methylated genes associated with aggressiveness in a small number of PR men with PCa [26]. Our findings on epigenetic differences between prostate tumor and adjacent non-involved (normal) tissues will be used to detect PCa early and may clarify how PCa starts from the normal tissues. In addition, our goal is to identify PR specific DNA methylation biomarkers responsible for cancer disparities in PR H/L men. In addition, we characterized the ancestry structure of PR patients who participated in this study. Via et al. analyzed 642 PR individuals for ancestry structure and reported that PR is an admixed population [27].

\section{Materials and Methods}

\subsection{IRB Approval, and Tissue Sample Selection}

Two Institutional Review Boards approved this study: the Moffitt Cancer Center (Protocol no. Pro00048100) and the Ponce Health Sciences University (PHSU) (Protocol no. 1909021277A001). All study participants signed an Informed Consent. We obtained 49 formalin-fixed paraffin-embedded (FFPE) prostate tumor and adjacent non-involved pair samples from the Puerto Rico Biobank (PRBB), a U54 PHSU-MCC PACHE Partnership core facility. Based on Gleason scores, tumors from study participants were classified as either aggressive, 17, or as indolent, 32.

\subsection{DNA Methylation Analysis}

\subsubsection{Illumina EPIC Methylation and DNA Samples}

We used the Illumina Infinium Methylation EPIC (EPIC) BeadChip DNA methylation platform to obtain DNA methylation levels in DNA samples from FFPE-preserved tissues as described in the manufacturer's instructions. This instrument is located at the Molecular Genomics Core, MCC, Tampa, FL, USA. Genomic DNA was obtained from the prostate tissues. DNA was extracted from the marked tumor area on the H\&E slides by the pathologist (J.D.). DNA quality was tested with DNA integrity numbers (DINs).

2.2.2. Quality Control and Normalization for Data Obtained from Epigenome-Wide Methylation Assays

Raw IDAT files were read by the minfi (version 1.28.4) $[28,29]$ Bioconductor package for $\mathrm{R}$ (version 3.5.2). Minfi's implementation detection $p$-value was used to calculate detection $p$-values. Normalization was performed using the normal-exponential out-of-band (NOOB) [30] method. Next, functional normalization (FunNorm) [31], a between-array normalization method, was used. As Illumina recommended, the preprocess Funnorm function returned an object containing $\beta$-values which were measured with an offset of 100 in the denominator [32]. To visualize data quality and identify outliers and potential batch effects, we used a histogram of $\beta$-values, the number of missing values, and principal component analysis (PCA). We set $\beta$-values with a corresponding detection $p$-value $>0.05$ as missing values.

\subsubsection{Detection of Differentially Methylated Regions (DMRs)}

Student's $t$-test and false discovery rates (FDR) were used for two group comparisons and multiple testing respectively [33]. We set the minimum point for the mean $\beta$-value between the two groups as 0.2. Therefore, $\mathrm{CpG}$ sites were considered significant if the difference in for tumor versus normal was $>0.2$ and 0.05 when comparing low-risk (indolent) and high-risk (aggressive) [34]. We used a region-based analysis to prevent potential false positives. We considered all the CpG probes within a specific gene and determined as DMRs if several CpG probes within that gene were differentially methylated [35-38]. We 
did not consider as a DMR if only one CpG probe was different within a specific gene. Statistical analysis was performed using MATLAB (Natick, MA, USA).

\subsection{Analysis for Ancestry Structure}

The ancestry informative markers (AIMs) were used to estimate ancestry structure of the 49 Puerto Rican men with the prostate cancer previously described. AIMs, 106 genetic variations, provide the proportion of indigenous American, African, and European ancestry. Genotyping was performed by a multiplex PCR coupled with single-base extension methodology using a Sequenom analyzer. We excluded 5 single nucleotide polymorphisms because of weak clustering or less than optimal genotyping rates $(<90 \%)$. Admixture [39] was used to estimate ancestry structure.

\subsection{Estimation of Epigenetic Mitotic Clocks}

The total number of stem cell divisions (TNSC) and stem cell division rate (SCDR) were calculated using epiTOC2 methods as described previously [25].

\section{Results}

\subsection{Demographic and Clinicopathological Characteristics of Study Group}

The mean age at diagnosis for PR H/L men in the two groups of PCa was 64.6 years for aggressive (high-risk) group, and 60.3 years for the indolent (low-risk) group. Thirty-five percent of all patients $(n=17)$ had a high Gleason score $(7(4+3)$ or $8-10)$ and were classified as a high-risk group. As expected, the significantly different distribution in clinical stage was detected between two groups. No statistically significant differences $(p>0.05)$ between the two groups were evident in terms of prostate-specific antigen (PSA) levels and surgical margins (Table 1).

Table 1. Clinicopathological characteristics of Puerto Rican men $(n=49)$ with prostate cancer in the pilot study.

\begin{tabular}{cccc}
\hline Risk & High & Low & p-Value \\
\cline { 2 - 3 } & $\boldsymbol{n}=\mathbf{1 7}$ & $\boldsymbol{n}=\mathbf{3 2}$ & 0.085 \\
Age at Diagnosis & $64.6 \pm 5.7$ & $60.3 \pm 9.1$ & 0.724 \\
PSA & $7.72 \pm 5.07$ & $7.01 \pm 6.46$ & $<0.0001$ \\
Gleason score & & & \\
6 & 0 & 20 & \\
$7(3+4)$ & 0 & 12 & $<0.0001$ \\
$7(4+3)$ & 11 & 0 & \\
$8-9$ & 6 & 0 & \\
Stage & 1 & 1 & \\
T1c & 2 & 9 & \\
T2a & 8 & 20 & \\
T2c & 1 & 1 & \\
T3a & 5 & 1 & \\
T3b & 1 & 3 & \\
Surgical margins & 14 & 27 & \\
Yes & 2 & 2 & \\
No & & & \\
Missing & & & \\
\hline$p$-values were obtained from Student's $t$ chi-square, or Fisher exact tests. PSA, prostate-specific antigen.
\end{tabular}

\subsection{Differential Methylation between Prostate Cancer Tumors and Adjacent Non-Involved Tissue}

Quality control analysis, PCA, $\beta$-value distributions, and missing values identified and excluded three samples (two tumors and one adjacent non-involved) which did not meet quality control criteria. The first principal component (PC1) showed a clear separation between tumor $(n=49)$ and adjacent non-involved tissues $(n=49)$ from the unsupervised PCA model based on the remaining DNA samples $(n=98)$ (Figure 1A). These data 
suggested significant differential methylation between adjacent non-involved and tumor tissues. To measure differential methylation levels between adjacent non-involved and tumor tissues, a two-group comparison was performed with a volcano plot. This is a type of scatterplot that shows statistical significance ( $q$-value) versus magnitude of change ( $\Delta \beta$-value). In this figure, the most hypermethylated $\mathrm{CpG}$ probes are towards the right, the most hypomethylated $\mathrm{CpG}$ probes are towards the left, and the most statistically significant genes are towards the top. A volcano plot was based on the false discovery rate (FDR)-corrected $p$-values $(q<0.001)$ and the mean difference $(\Delta \beta$-value $>0.2)$ between the two groups (Figure 1B). From this analysis, we identified 8293 differentially methylated CpG probes; the majority of probes $(n=7744)$ were hypermethylated in tumor samples (Figure 1B). A scatter density graph of the average $\beta$ value for tumor vs. adjacent normal samples demonstrated that many CpG-probes have a similar methylation level in tumor and normal samples, since most of the CpG-probes are along the diagonal (Figure 1C). The "bump" located at $x=0.24, y=0.5$ indicates an increase of methylation in PCa tumor samples. A histogram of the $\Delta \beta$-value showed more hyper-methylated probes than hypo-methylated probes, especially for $\Delta \beta$-value $>0.2$ (Figure 1D).

With the DAVID Functional Annotation Tools [40], we grouped genes based on functional similarity in order to better interpret the large lists of genes derived from epigenomewide analysis. From 12 functional annotation categories, the highest enriched gene-set for hyper-methylated probes was the Homeobox group (Figure 1E). We compared the $\Delta \beta$ value for significant hyper-methylated $\mathrm{CpG}$-probes based on functional locations, DNase Hypersensitivity CpG-probes (DHS), Open Chromatin probes (OC), and Transcription factor binding sites (TFBS) (Figure 1F).

Based on DMR selection criteria, we identified 3034 differential probes in 892 genes. Some identified genes, such GSTP1 (Figure 2A), RARB (Figure 2B), and RASSF1 (Figure 2C), which were previously suggested as methylation biomarkers for PCa [14], showed DMRs in tumor tissues. In addition, some genes, including Tumor Protein D52 (TPD52), which is an oncogene [41], showed hypomethylation in multiple CpG sites in prostate tumor tissues (Figure 2D).

Hypermethylation of $R A R B$ showed a significant association with PCa risk (OR 1.76, 95\% CI: 1.29-2.40), and the association was more evident in NHBs (OR 2.18, 95\% CI: 1.39-3.44) [40]. A role of methylation in RASSF1A gene in PCa risk was reported in a meta-analysis. The odds ratio (OR) of RASSF1A methylation in men with PCa, compared to controls, was 14.7 (95\% CI = 7.6-28.6), with high specificity (AUC: 0.87, 95\% CI: 0.72-0.94) and sensitivity (AUC: 0.76, 95\% CI: 0.55-0.89). Therefore, promoter methylation of the RASSF1A gene has been suggested as a potential biomarker for PCa risk [41]. Additionally, the hypomethylated genes in this study, such as tumor protein D52 (TPD52), an oncogene, have been reported to be over-expressed in PCa as compared with adjacent normal tissues. Therefore, methylation in TPD52 was proposed as a biomarker for PCa risk [42]. These results can be further evaluated for their contribution to PCa risk in PR PCa patients. 
A

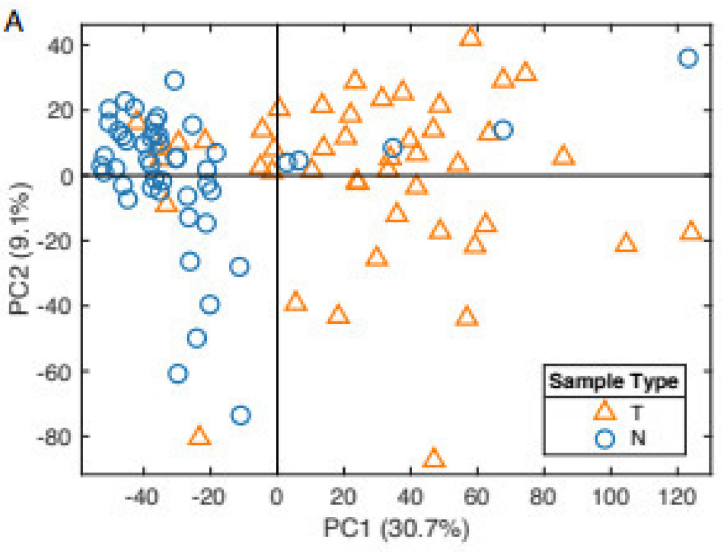

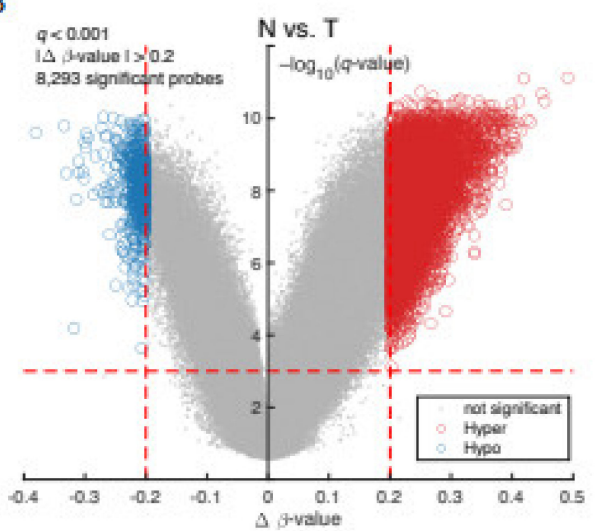

C

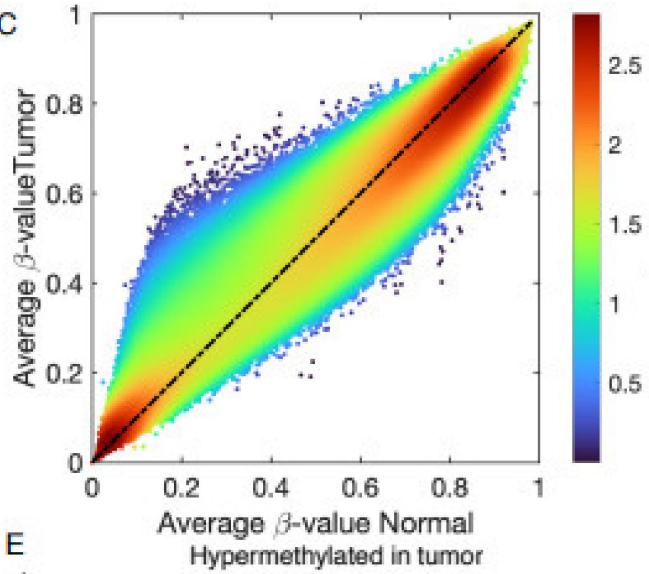

D

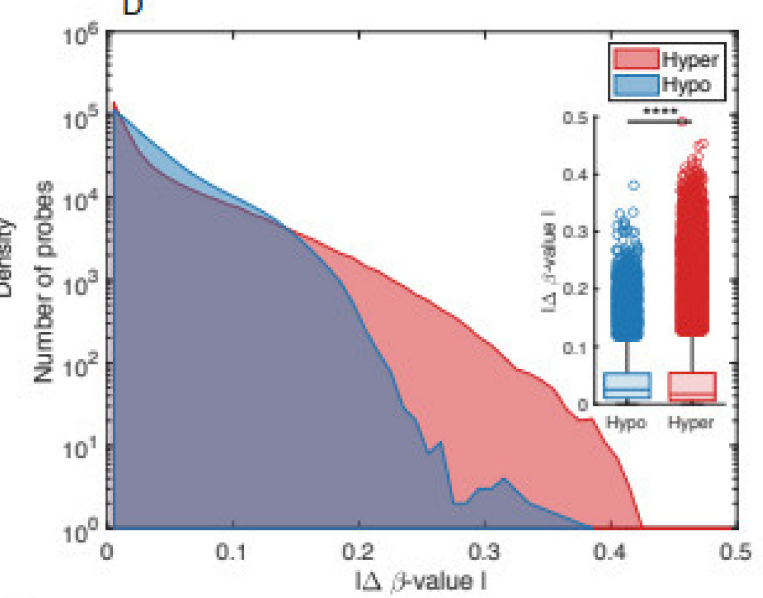

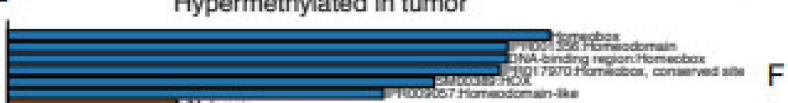

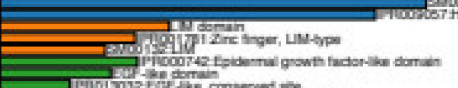

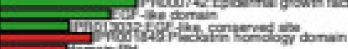

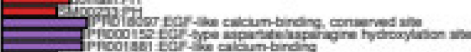

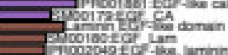

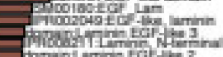

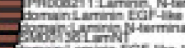

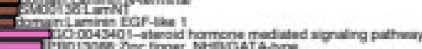

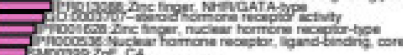

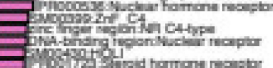

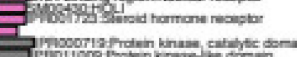

Fringoger

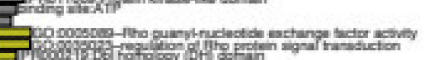

Z

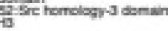

ב.

寻

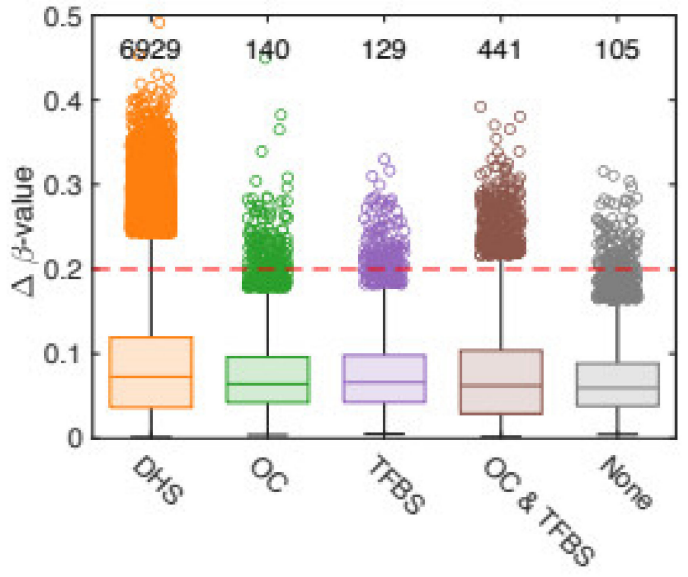

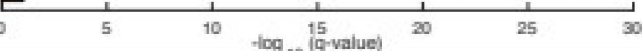

Figure 1. Analysis of tumor vs. normal tissues. The two first principal components in a PCA model using all 49 tumor and 49 adjacent normal samples and all CpG-probes $(n=807,583)$ separates normal (blue circles) and tumor (orange triangles) tissues from each other (A). A volcano plot comparing tumor vs. normal tissues with $\Delta \beta$-value on the $x$-axis and FDR corrected $p$-value, $-\log _{10}(q$-value), on the $y$-axis (B). Blue circles indicate significant hypo-methylated CpG-probes and red circles hypermethylated CpG-probes. Scatter density graph of average $\beta$-value for tumor samples vs. normal samples (C). Histogram comparing the number of hypo- and hyper-methylated probes (D). Enriched gene-sets for hyper-methylated probes using DAVID (E). Comparing the $\Delta \beta$-value for significant hyper-methylated CpG-probes (F). DNase Hypersensitivity CpG-probes (DHS), Open Chromatin probes (OC), Transcription factor binding sites (TFBS). ${ }^{* * * *} p<0.0001$. 
A

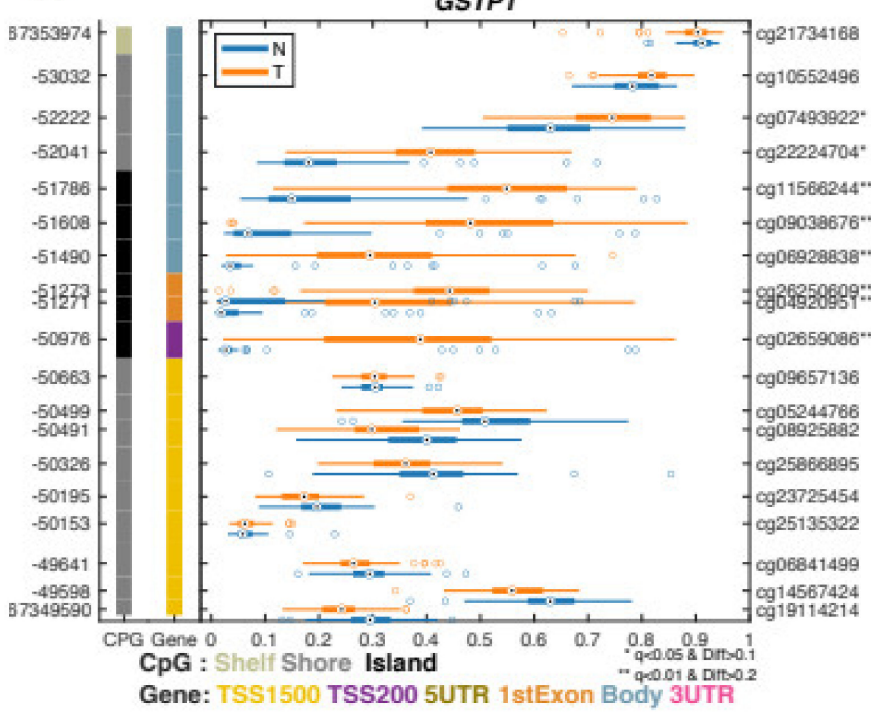

B

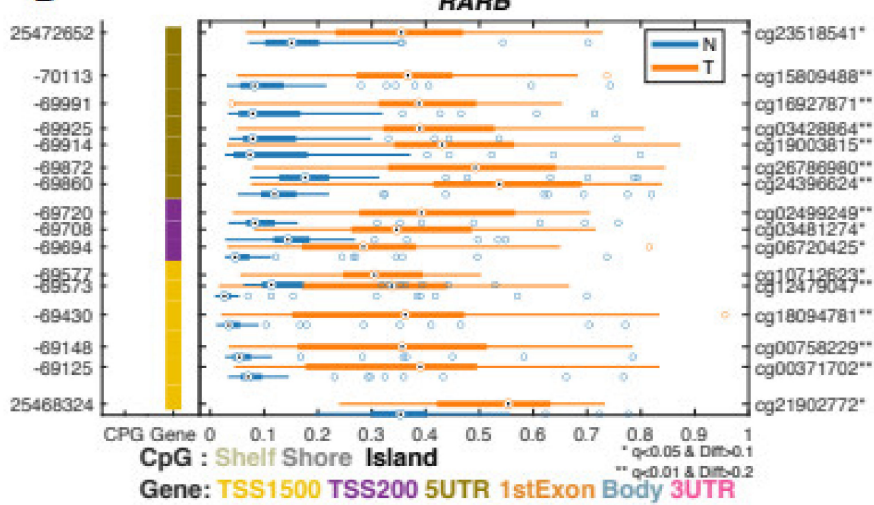

C

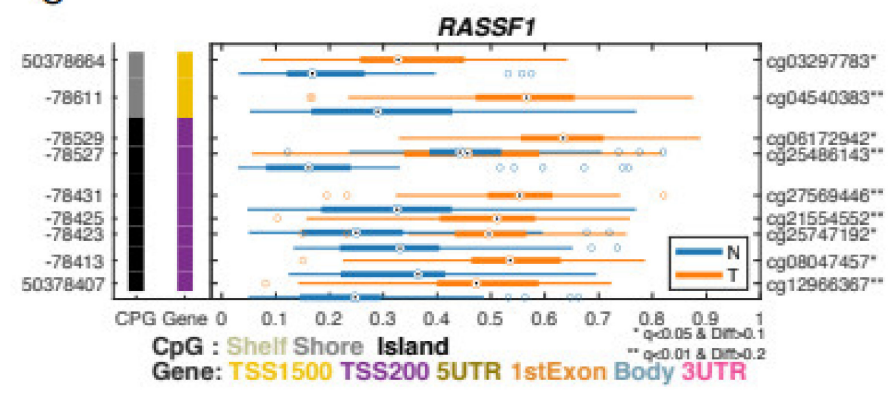

D

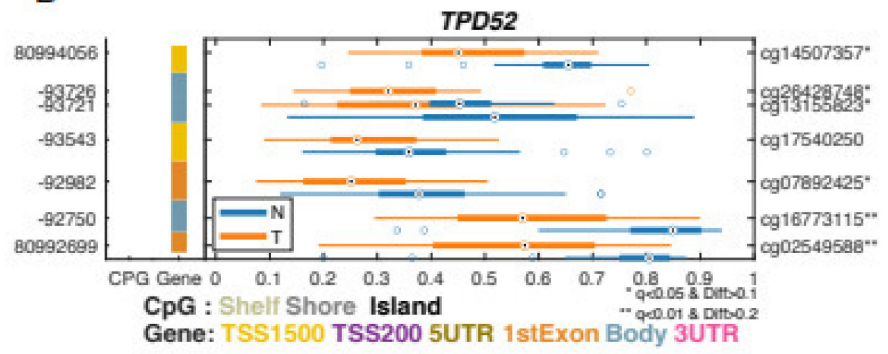

$\mathrm{E}$

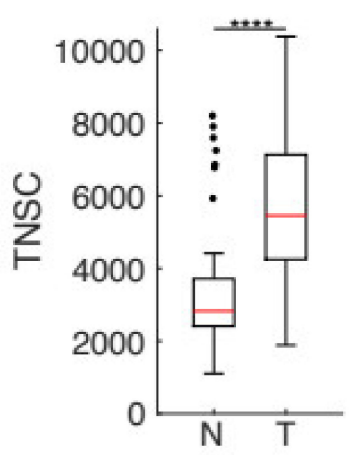

$\mathrm{F}$

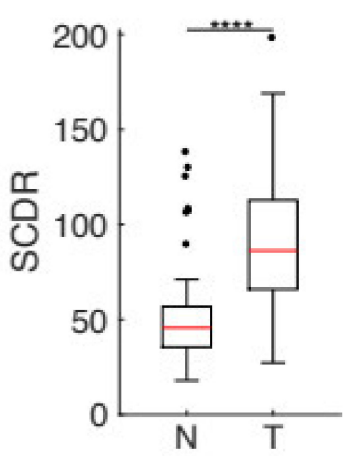

Figure 2. Differently methylated genes in tumor vs. normal tissues. GSM plots comparing the methylation levels between normal and tumor tissues for GSTP1 (A), RARB (B), RASSF1 (C), and TPD52 (D). The selected CpG probes are shown on the $y$-axis with probe-id on the right $y$-axis and the genomic position on the left $y$-axis. The methylation level for each $\mathrm{CpG}$ probe is shown along the $x$-axis ( $\beta$-value) with a boxplot for normal samples $(\mathrm{N})$ in blue and tumor samples $(\mathrm{T})$ in orange. The leftmost column indicated $\mathrm{CpG}$ island while the second column indicates the CpG probes location in the gene. Total number of stem cell divisions per stem cell (TNSC) boxplot comparing tumor vs. normal tissues (E), and stem cell division rate (SCDR) boxplot (F). ${ }^{* * * *} p<0.0001$.

\subsection{Comparison of Epigenetic Clocks between Tumor vs. Adjacent Normal Tissues}

Risk of mutations in a cell are increased by a high turnover rate, and this is associated with an accelerated cell division as part of the cell cycle [21]. Therefore, the mitotic age is correlated with the risk of carcinogenesis [19]. With Tischendorf's epigenetic mitotic clocks [42], we estimated a mathematical expression to estimate the fraction of cells methylated at 163 candidate $\mathrm{CpG}$ sites in tumor or adjacent normal tissues. The total number of stem cell divisions (TNSC) at the patient's age, and the parameters for prostate-specific probability of de novo methylation and baseline methylation (i.e., at fetal stage) were determined. We note that TNSC and stem cell division rate (SCDR) in tumor tissues were significantly higher than adjacent normal tissues $(p<0.0001)$ (Figure 2E,F). 


\subsection{Differentially Methylated Genes Associated with Aggressive Type of Prostate Cancer}

To further investigate the differentially methylated genes in prostate tumor samples, we calculated a PCA model using only the tumor samples. The unsupervised PCA model did not show a clear distinction between tumor and adjacent non-involved tissues in the first principal component (PC1) (Figure 3A).

A

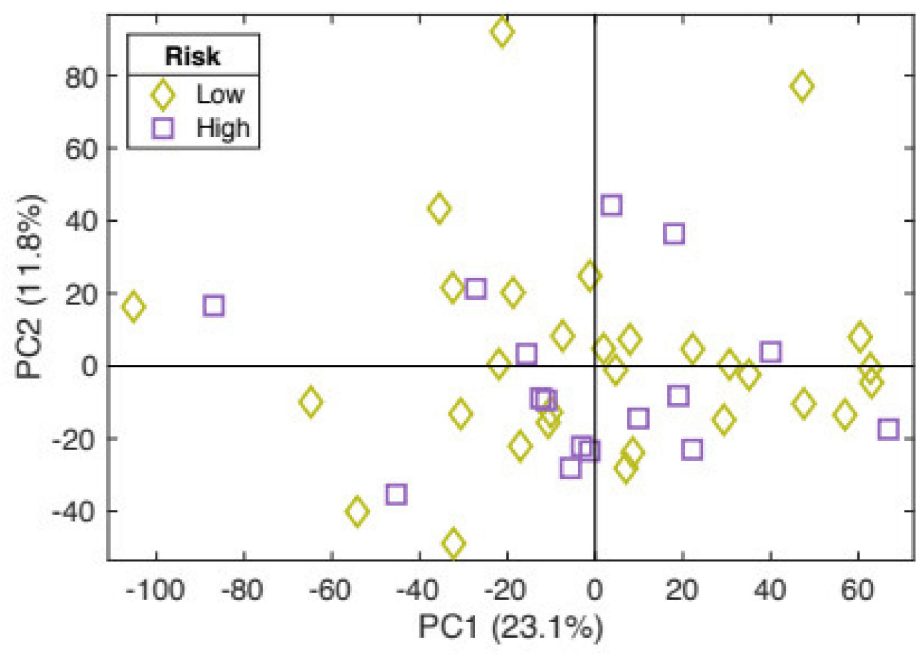

C

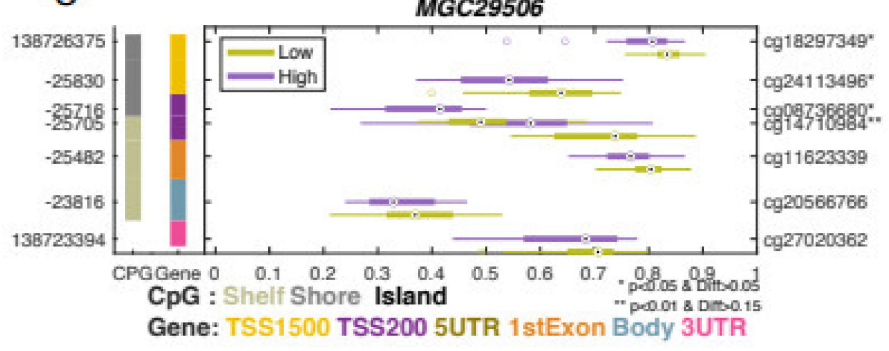

B

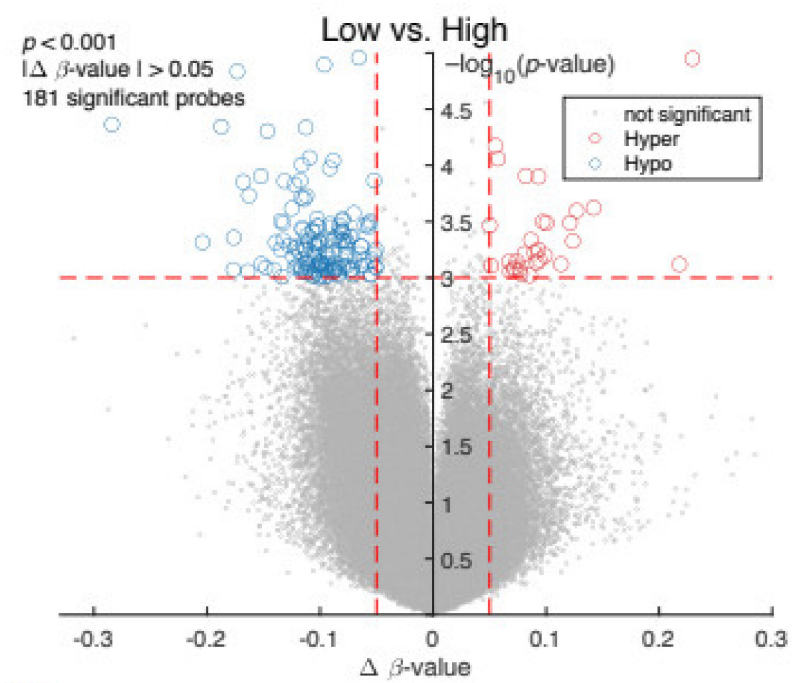

D

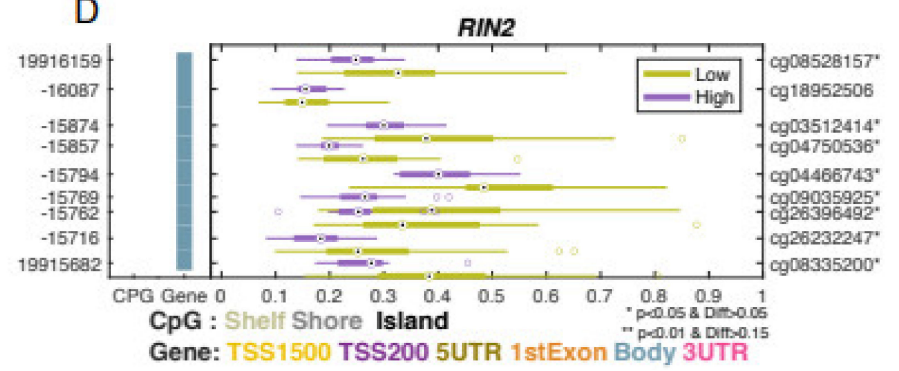

Figure 3. Analysis of high-risk vs. low risk. The two first principal components in a PCA model using all tumor samples $(n=49)$ and all CpG-probes $(n=785,071)$ with low-risk tumors (green diamonds) and high-risk tumors (purple squares) (A). A volcano plot comparing low-risk tumors vs. high-risk tumors with $\Delta \beta$-value on the $x$-axis and $p$-value, $-\log _{10}(p$-value), on the $y$-axis (B). Blue circles indicate significant hypo-methylates $\mathrm{CpG}$-probes and red circles hyper-methylated CpG-probes. GSM plots comparing the methylation levels between low-risk tumors and high-risk tumors for MGC29506 (C), RIN2 (D).

The $p$ values $(p<0.001)$ and the mean difference $(\Delta \beta$-value $>0.05)$ between the indo-lent and aggressive groups are presented in a volcano plot (Figure $3 \mathrm{~B}$ ). This analysis resulted in 181 differentially methylated $\mathrm{CpG}$ probes, with a majority of the probes showing hypomethylation in tumor samples (Figure 3B). The volcano plot shows the significant differentially methylated $\mathrm{CpG}$ sites between aggressive and indolent prostate tumors found in 141 genes (Figure 3B). Supplementary Table S1 presents the gene symbol, $p$-values, probeID, and mean difference of methylation levels in high- and low-risk groups. Some identified genes include RIN2 (Figure 3D), which was previously suggested as a methylation biomarker for early stage of esophageal cancer [43], and MGC29506 (Figure 3C), which was previously suggested as a biomarker for gastric [44] and testicular cancers [23].

Among 181 differently methylated CpG sites, methylation level was significantly increased in 130 sites and significantly decreased in 51 sites, in 141 unique genes $(p<0.001)$, and the strongest evidence was found for SPRED2 [43], HLA-C [44], TMEM108 [45], and PRKAG2 [46], which are involved in MARK signaling, immune pathway, and cellular homeostasis. 


\subsection{Ancestry Analysis}

We determined the ancestry structure for the 49 study participants. The contribution of African ancestry ranged between $<1 \%$ and $85.3 \%$, averaging $21.9 \%$ (standard deviation, SD 17.8\%) (Figure 4 and Table 2). We found that the European and indigenous American ancestry components were $65.8 \%$ and $12.3 \%$, on average, respectively. Interestingly, in Puerto Rican men in this study, there were large variations in European and African ancestries. However, the Indigenous American ancestries were relatively homogenous (Table 2, Figure 4).

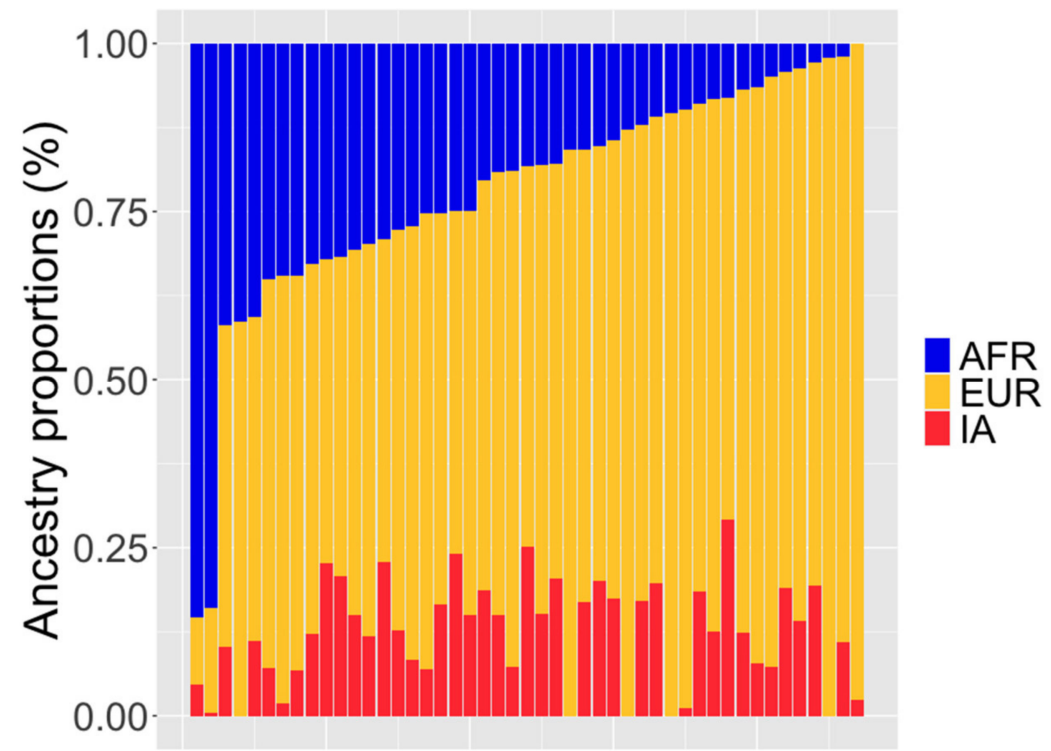

Figure 4. Visualization of the ancestry proportions for each individual in $49 \mathrm{PR} \mathrm{H} / \mathrm{L}$ men with PCa (Puerto Rico PCa) compared to 1000 Genomes Admixed Americans populations. Assuming three ancestral populations $(k=3)$, each column represents one individual, and each color corresponds to the contribution of each ancestral population to the genome of a given individual (blue = African, yellow $=$ European, and red = Indigenous American).

Table 2. Ancestry proportions in the study cohort $(n=49)$.

\begin{tabular}{ccccc}
\hline Ancestral Population & Average & SD & Maximum & Minimum \\
\hline African & 0.219 & 0.178 & 0.853 & 0.00001 \\
European & 0.658 & 0.179 & 0.978 & 0.0995 \\
Indigenous American & 0.123 & 0.0784 & 0.292 & 0.00001 \\
\hline
\end{tabular}

SD: standard deviation.

\section{Discussion}

Puerto Rican Hispanic/Latino men are at an increased risk of prostate cancer-specific mortality compared with NHW men. However, the relationship between this observation and epigenetics and how this relationship explains PCa racial and ethnic health disparities are controversial [26]. This study represents an ongoing effort to investigate DNA methylation in PR H/L men with PCa. We observed that several differentially methylated genes were found in aggressive tumor tissue from PR PCa patients. Our findings will help us to both understand why and know when PR patients have an aggressive type of prostate cancer. Once we identify these unique DNA methylation patterns at the time the patient is diagnosed, long-term, they may provide new molecular tools to clinicians to determine their treatments for PR H/L men with PCa with high risk.

This study represents the first steps towards implementing personalized medicine in PR PCa patients. This approach is tailored to the biology of the individual, making it more effective than a "one size fits all" approach. It may provide a molecular tool to clinicians to reduce cancer health disparities. 
We identified 892 genes that showed significant differential methylation for multiple probes in the prostate tumor tissue. The promising methylation marker candidates were identified in many genes including: GSTP1, RARB, RASSF1, and TPD52 (Figure 2). GSTP1 is one of the metabolizing enzymes and plays a key role in preventing the carcinogenesis caused by environmental exposures [47]. Hypermethylation of the GSTP1, a tumor suppressor gene, frequently occurs in different cancer types, including PCa [48]. Hypermethylation of the GSTP1 is associated with down-regulation of GSTP1 expression, and eventually leads to carcinogenesis. Therefore, hypermethylation of GSTP1 was suggested as a biomarker of early stage of PCa [49]. Interestingly, GSTP1 methylation in tumors is more strongly related to NHB men with PCa [50] than NHWs. RARB is one of the well-known tumor suppressor genes, frequently hypermethylated in prostate tumorigenesis [51].

Massie et al. (2017) reviewed associations with DNA methylations and PCa and reported a list of frequently differentially methylated genes in prostate tumor tissues [7]. After an extensive review of 17 studies, they identified 5962 differentially methylated genes in tumors. Among 892 genes we identified, 526 (59.0\%) genes, including GSTP1 and SMAD3, were validated in the list of genes from Massie's review.

Methylated genes $(n=366)$ identified in this study were not found in the list of Massie's study. This may be partly due to differences in methodology, platforms used, sample size, and the unique ancestral composition of the Puerto Rican population, among others, or can be specific to the PR patients studied. These genes observed in this study may contribute to differences in PCa outcomes that have been reported between PR and NHW men. DNA methylation continues to change during progression of PCa with different characteristics at different stages if the disease. Therefore, it is crucial to study the epigenetic clock or DNA methylation age (DNAmAge) to understand a picture of the epigenetic landscape in PCa progression.

Several groups have reported different methylation patterns between PCa tumor and adjacent normal tissues [52]. Using epigenome-wide DNA methylation data derived from 73 PCa tumors and 63 adjacent prostatic tissue samples, Kirby et al. (2017) identified methylation patterns that distinguished prostate tumor from adjacent normal tissue with a high predictive power [53]. However, this study only compared tumors with adjacent normal tissues, and different methylation pattens in aggressive PCa were not assessed. We identified 141 differentially methylated genes, including MGC29506 and RIN2, after comparison of PR patients with aggressive vs. indolent PCa (Supplementary Table S1).

Currently, the exact function of the MGC29506 gene is unknown. Katoh and Katoh (2003) reported that the expression of MGC29506 was down-regulated in intestinal-type gastric cancer as compared with adjacent normal tissues [54]. Further, MGC29506 protein inhibited proliferation of cells by arresting cells at the G0/G1 and S phases of the cell cycle. These results suggested the potential of MGC29506 as a suppressor gene in gastric cancer [55].

Although RIN2 has not been studied in terms of its role in PCa, as one of DNA damage and repair related gene sets, this gene has been investigated in colorectal [56] and esophageal [57] cancers. Recently Wang et al. (2021) reported a 12-gene-based prognostic signature selected from 160 DNA damage and repair related genes. This signature can predict survival of patients with colorectal cancer (AUC: 0.80) [56]. Alvi et al. (2013) reported that methylation status of four genes, including RIN2, can distinguish between esophageal tumor and benign tissues with high accuracy (AUC: 0.98) [57].

We identified several genes associated with prostate cancer. One of genes we identified is PRDM16. Few studies have reported for the role of PRDM16 in PCa. Chandrashekar et al. reported that PRDM16 expression was associated with the survival of PCa patients with different Gleason scores. Its expression in prostate tumor indicated a high diagnostic value for early detection of PCa [58]. Another gene, TP73 were extensively investigated in prostate cancer. We previously reported a significant role of genetic variation in TP73 in prostate cancer. We detected a significant inverse relationship between p73 variation and 
PCa aggressiveness. Additionally, p73 variation is marginally associated with overall death as well as PCa-specific death [59].

SPRED2 is known as one of the key negative regulators of the MAPK signaling pathway. Kachroo et al. observed the downregulation of SPRED2 in an aggressive type of prostate cancer and SPRED2 overexpression suppressed prostate cancer cell proliferation [43]. Regulation of HLA expression on cell surface is involved in natural killer cell-medicated lysis of tumor cells [44]. A role of HLA genes was investigated in cervical cancer. HLA-C group 1 was significantly more transmitted with invasive cervical cancer [60]. PRKAG2 encodes a subunit of AMP-activated protein kinase (AMPK), which is a cellular homeostasis sensor. Sakabe et al. reported that PRKAG2 expression was correlated with survival in liver cancer patients with IFN-a/5-FU treatment [46]. Similar results were observed in liver and lung cancer patients [61,62]. TMEM108 may have a role in IFN signaling through the Wnt-b-catenin pathway. eQTL analyses suggested that the mechanism of THEM108 is through the immune infiltrating cells and adjacent non-involved tissue around the tumor [45].

This study has strengths and limitations. The first strength is that it is the largest study of epigenome-wide DNA methylation profiles of PR PCa patients. We previously reported results of epigenome-wide methylation analysis based on 24 PR PCa cases [26]. The second strength is that the identification of DNA methylation profiles associated with risk and aggressiveness of PCa in PR H/L men will provide a potential mechanism for studying PCa disparities in the PR population and to generate hypotheses for future studies. Apprey et al. (2019) reported significant different methylation profiles between racial groups [18]. We are currently investigating whether the ancestry proportions influence the methylation patterns in PR H/L men with PCa. As limitations, the sample size of this study, especially for the aggressive PCa phenotype, was small. Therefore, this study lacked enough statistical power for broad generalizations. In addition, the lack of an independent validation set for confirmation of our findings is another limitation factor. In future, we plan to confirm our findings in cfDNA or DNA from urine or blood of PCa patients. This approach with minimal invasive methods may represent an important contribution in the clinical management of these patients.

In summary, we identified 141 differentially methylated genes in tumor tissues from PR PCa patients with the aggressive phenotype. Although our findings still need further validation, they provide an important insight into the epigenetic landscape of prostate cancer in the PR H/L patient population. Some of the genes identified in this study were associated with various cancers, including PCa, and affect various biological processes, such as immune pathways, cell signaling, metabolism, DNA repair, proliferation, and cell cycle.

Supplementary Materials: The following are available online at https:/ / www.mdpi.com/article/ 10.3390/biom12010002/s1, Table S1: Differentially methylated genes between aggressive (high-risk) and indolent (low-risk) prostate tumors in Puerto Rican men with prostate cancer.

Author Contributions: Conceptualization, G.R.-D., J.M. (Jaime Matta), J.E.-M., C.O.-S. and J.Y.P.; data curation, J.M. (Jaime Matta), J.E.-M., C.O.-S., J.M. (Joshua Marcial), C.A.-T., R.P., N.M., J.D. (Jasreman Dhillon) and R.L.; formal analysis, J.D. (Julie Dutil), A.B., H.-Y.L., R.P. and Y.K.; funding acquisition, G.R.-D., J.M. (Jaime Matta) and J.Y.P.; investigation, G.R.-D., J.M. (Jaime Matta), J.E.-M., C.O.-S., R.C., K.Y., C.D.O., L.W., N.M., J.D. (Julie Dutil), S.J.K., J.D. (Jasreman Dhillon) and J.Y.P.; methodology, J.E.-M., C.O.-S., R.P., A.B., J.D. (Julie Dutil) and J.Y.P.; visualization, A.B. and J.D. (Julie Dutil); project administration, J.Y.P.; writing — original draft, G.R.-D., J.M. (Jaime Matta) and J.Y.P.; writing-review and editing, A.B., G.R.-D., J.M. (Jaime Matta), J.E.-M., C.O.-S., J.D. (Julie Dutil), R.L., J.M. (Joshua Marcial), C.A.-T., R.P., R.C., C.D.O., H.-Y.L., K.Y., N.M., J.D. (Jasreman Dhillon), L.W., Y.K., S.J.K. and J.Y.P. All authors have read and agreed to the published version of the manuscript.

Funding: This study including the Puerto Rico Biobank and Quantitative Sciences core were supported by the National Cancer Institute of the National Institutes of Health, NCI Center to Reduce Health Disparities with grants awarded to Ponce Health Sciences University (PHSU) and PHSUMoffitt Cancer Center Partnership grants 5U54CA163071 and U54 CA163068. This work was partially 
supported by the Dept of Defense (DoD), through the prostate cancer research program (PCRP) under Award No. W81XWH2110241.

Institutional Review Board Statement: The study was conducted according to the guidelines of the Declaration of Helsinki and approved by the Institutional Review Board of Ponce Health Sciences University (Protocol code 1909021277A001, approved on 24 April 2019) and of the Moffitt Cancer Center (Protocol no. Pro00048100).

Informed Consent Statement: Informed consent was obtained from all subjects involved in the study.

Data Availability Statement: The data presented in this study are available on request from the corresponding author. The data are not publicly available due to privacy of participants.

Acknowledgments: The authors acknowledge supports from the Quantitative Sciences Core (QSC), the Puerto Rico Biobank (PRBB) and the financial support from grants \# U54 CA163071 and U54 CA163068-07. This work has been supported in part by the Biostatistics and Bioinformatics Shared Resource at the H. Lee Moffitt Cancer Center \& Research Institute, an NCI designated Comprehensive Cancer Center (P30-CA076292).

Conflicts of Interest: The authors declare no conflict of interest.

\section{References}

1. ACS. Cancer Facts \& Figures 2021; American Cancer Society: Atlanta, GA, USA, 2021.

2. NCI. Cancer Stat Facts: Prostate Cancer; Surveillance, Epidemiology, and End Results Program; National Cancer Institute: Bethesda, MD, USA, 2018.

3. Chinea, F.M.; Patel, V.N.; Kwon, D.; Lamichhane, N.; Lopez, C.; Punnen, S.; Kobetz, E.N.; Abramowitz, M.C.; Pollack, A. Ethnic heterogeneity and prostate cancer mortality in Hispanic/Latino men: A population-based study. Oncotarget 2017, 8, 69709-69721. [CrossRef]

4. Tortolero-Luna, G.; Zavala-Zegarra, D.; Pérez-Ríos, N.; Torres-Cintrón, C.R.; Ortiz-Ortiz, K.J.; Traverso-Ortiz, M.; Román-Ruiz, Y.; Veguilla-Rosario, I.; Vázquez-Cubano, N.; Merced-Vélez, M.F.; et al. Cancer in Puerto Rico, 2006-2010; Puerto Rico Central Cancer Registry: San Juan, PR, USA, 2013.

5. Mateo, J.; Carreira, S.; Sandhu, S.; Miranda, S.; Mossop, H.; Perez-Lopez, R.; Rodrigues, D.N.; Robinson, D.; Omlin, A.; Tunariu, N.; et al. DNA-Repair Defects and Olaparib in Metastatic Prostate Cancer. N. Engl. J. Med. 2015, 373, 1697-1708. [CrossRef]

6. Xu, N.; Wu, Y.P.; Ke, Z.B.; Liang, Y.C.; Cai, H.; Su, W.T.; Tao, X.; Chen, S.H.; Zheng, Q.S.; Wei, Y.; et al. Identification of key DNA methylation-driven genes in prostate adenocarcinoma: An integrative analysis of TCGA methylation data. J. Transl. Med. 2019, 17, 311. [CrossRef] [PubMed]

7. Massie, C.E.; Mills, I.G.; Lynch, A.G. The importance of DNA methylation in prostate cancer development. J. Steroid Biochem. Mol. Biol. 2017, 166, 1-15. [CrossRef]

8. Cancer Genome Atlas Research. The molecular taxonomy of primary prostate cancer. Cell 2015, 163, 1011-1025. [CrossRef] [PubMed]

9. Carleton, N.M.; Zhu, G.; Gorbounov, M.; Miller, M.C.; Pienta, K.J.; Resar, L.M.S.; Veltri, R.W. PBOV1 as a potential biomarker for more advanced prostate cancer based on protein and digital histomorphometric analysis. Prostate 2018, 78, 547-559. [CrossRef] [PubMed]

10. Komura, K.; Sweeney, C.J.; Inamoto, T.; Ibuki, N.; Azuma, H.; Kantoff, P.W. Current treatment strategies for advanced prostate cancer. Int. J. Urol. 2018, 25, 220-231. [CrossRef] [PubMed]

11. Giudice, A.; Montella, M.; Boccellino, M.; Crispo, A.; D’Arena, G.; Bimonte, S.; Facchini, G.; Ciliberto, G.; Botti, G.; Quagliuolo, L.; et al. Epigenetic changes induced by green tea catechins are associated with prostate cancer. Curr. Mol. Med. 2017, 17, 405-420. [CrossRef]

12. Pan, H.; Renaud, L.; Chaligne, R.; Bloehdorn, J.; Tausch, E.; Mertens, D.; Fink, A.M.; Fischer, K.; Zhang, C.; Betel, D.; et al. Discovery of Candidate DNA Methylation Cancer Driver Genes. Cancer Discov. 2021, 11, 2266-2281. [CrossRef] [PubMed]

13. Park, J.Y. Promoter hypermethylation in prostate cancer. Cancer Control 2010, 17, 245-255. [CrossRef] [PubMed]

14. Yang, M.; Park, J.Y. DNA methylation in promoter region as biomarkers in prostate cancer. Methods Mol. Biol. 2012, 863, 67-109. [PubMed]

15. Devaney, J.M.; Wang, S.; Funda, S.; Long, J.; Taghipour, D.J.; Tbaishat, R.; Furbert-Harris, P.; Ittmann, M.; Kwabi-Addo, B. Identification of novel DNA-methylated genes that correlate with human prostate cancer and high-grade prostatic intraepithelial neoplasia. Prostate Cancer Prostatic Dis. 2013, 16, 292-300. [CrossRef] [PubMed]

16. Kim, J.W.; Kim, S.-T.; Turner, A.R.; Young, T.; Smith, S.; Liu, W.; Lindberg, J.; Egevad, L.; Gronberg, H.; Isaacs, W.B.; et al. Identification of new differentially methylated genes that have potential functional consequences in prostate cancer. PLoS ONE 2012, 7, e48455. [CrossRef] [PubMed] 
17. Geybels, M.S.; Zhao, S.; Wong, C.-J.; Bibikova, M.; Klotzle, B.; Wu, M.; Ostrander, E.A.; Fan, J.-B.; Feng, Z.; Stanford, J.L. Epigenomic profiling of DNA methylation in paired prostate cancer versus adjacent benign tissue. Prostate 2015, 75, 1941-1950. [CrossRef]

18. Apprey, V.; Wang, S.; Tang, W.; Kittles, R.A.; Southerland, W.M.; Ittmann, M.; Kwabi-Addo, B. Association of genetic ancestry with DNA methylation changes in prostate cancer disparity. Anticancer Res. 2019, 39, 5861-5866. [CrossRef] [PubMed]

19. Tomasetti, C.; Vogelstein, B. Cancer etiology. Variation in cancer risk among tissues can be explained by the number of stem cell divisions. Science 2015, 347, 78-81. [CrossRef] [PubMed]

20. Issa, J.P. Aging and epigenetic drift: A vicious cycle. J. Clin. Investig. 2014, 124, 24-29. [CrossRef] [PubMed]

21. Baylin, S.B.; Ohm, J.E. Epigenetic gene silencing in cancer-A mechanism for early oncogenic pathway addiction? Nat. Rev. Cancer 2006, 6, 107-116. [CrossRef] [PubMed]

22. Slieker, R.C.; BIOS Consortium; Van Iterson, M.; Luijk, R.; Beekman, M.; Zhernakova, D.V.; Moed, M.H.; Mei, H.; Van Galen, M.; Deelen, P.; et al. Age-related accrual of methylomic variability is linked to fundamental ageing mechanisms. Genome Biol. 2016, 17, 109. [CrossRef]

23. Hannum, G.; Guinney, J.; Zhao, L.; Zhang, L.; Hughes, G.; Sadda, S.; Klotzle, B.; Bibikova, M.; Fan, J.-B.; Gao, Y.; et al. Genome-wide methylation profiles reveal quantitative views of human aging rates. Mol. Cell 2013, 49, 359-367. [CrossRef]

24. Dugué, P.-A.; Bassett, J.K.; Joo, J.E.; Jung, C.-H.; Wong, E.M.; Moreno-Betancur, M.; Schmidt, D.; Makalic, E.; Li, S.; Severi, G.; et al. DNA methylation-based biological aging and cancer risk and survival: Pooled analysis of seven prospective studies. Int. J. Cancer 2018, 142, 1611-1619. [CrossRef]

25. Teschendorff, A.E. A comparison of epigenetic mitotic-like clocks for cancer risk prediction. Genome Med. 2020, 12, 56. [CrossRef] [PubMed]

26. Ruiz-Deya, G.; Matta, J.; Encarnación-Medina, J.; Ortiz-Sanchéz, C.; Dutil, J.; Putney, R.; Berglund, A.; Dhillon, J.; Kim, Y.; Park, J.Y. Differential DNA methylation in prostate tumors from Puerto Rican men. Int. J. Mol. Sci. 2021, 22, 733. [CrossRef] [PubMed]

27. Via, M.; Gignoux, C.R.; Roth, L.A.; Fejerman, L.; Galanter, J.; Choudhry, S.; Toro-Labrador, G.; Viera-Vera, J.; Oleksyk, T.K.; Beckman, K.; et al. History shaped the geographic distribution of genomic admixture on the island of Puerto Rico. PLoS ONE 2011, 6, e16513. [CrossRef] [PubMed]

28. Aryee, M.J.; Jaffe, A.E.; Corrada-Bravo, H.; Ladd-Acosta, C.; Feinberg, A.P.; Hansen, K.D.; Irizarry, R.A. Minfi: A flexible and comprehensive Bioconductor package for the analysis of Infinium DNA methylation microarrays. Bioinformatics 2014, 30, 1363-1369. [CrossRef]

29. Fortin, J.-P.; Triche, T.J., Jr.; Hansen, K.D. Preprocessing, normalization and integration of the Illumina HumanMethylationEPIC array with minfi. Bioinformatics 2016, 33, 558-560. [CrossRef] [PubMed]

30. Triche, T.J., Jr.; Weisenberger, D.J.; van den Berg, D.; Laird, P.W.; Siegmund, K.D. Low-level processing of Illumina Infinium DNA Methylation BeadArrays. Nucleic Acids Res. 2013, 41, e90. [CrossRef] [PubMed]

31. Fortin, J.-P.; Labbe, A.; Lemire, M.; Zanke, B.W.; Hudson, T.J.; Fertig, E.J.; Greenwood, C.M.T.; Hansen, K.D. Functional normalization of 450k methylation array data improves replication in large cancer studies. Genome Biol. 2014, 15, 503. [CrossRef]

32. Du, P.; Zhang, X.; Huang, C.-C.; Jafari, N.; Kibbe, W.A.; Hou, L.; Lin, S.M. Comparison of Beta-value and M-value methods for quantifying methylation levels by microarray analysis. BMC Bioinform. 2010, 11, 587. [CrossRef] [PubMed]

33. Johnson, W.E.; Li, C.; Rabinovic, A. Adjusting batch effects in microarray expression data using empirical Bayes methods. Biostatistics 2006, 8, 118-127. [CrossRef]

34. Wilhelm-Benartzi, C.S.; Koestler, D.C.; Karagas, M.R.; Flanagan, J.M.; Christensen, B.C.; Kelsey, K.T.; Marsit, C.J.; Houseman, E.A.; Brown, R. Review of processing and analysis methods for DNA methylation array data. Br. J. Cancer 2013, 109, $1394-1402$. [CrossRef] [PubMed]

35. Jaffe, A.E.; Murakami, P.; Lee, H.; Leek, J.T.; Fallin, M.D.; Feinberg, A.P.; Irizarry, R.A. Bump hunting to identify differentially methylated regions in epigenetic epidemiology studies. Int. J. Epidemiol. 2012, 41, 200-209. [CrossRef] [PubMed]

36. Siegel, E.M.; Berglund, A.E.; Riggs, B.M.; Eschrich, S.A.; Putney, R.M.; Ajidahun, A.O.; Coppola, D.; Shibata, D. Expanding epigenomics to archived FFPE tissues: An evaluation of DNA repair methodologies. Cancer Epidemiol. Biomark. Prev. 2014, 23, 2622-2631. [CrossRef]

37. Siegel, E.M.; Eschrich, S.; Winter, K.; Riggs, B.; Berglund, A.; Ajidahun, A.; Simko, J.; Moughan, J.; Ajani, J.; Magliocco, A.; et al. Epigenomic characterization of locally advanced anal. cancer: A radiation therapy oncology group 98-11 specimen study. Dis. Colon Rectum 2014, 57, 941-957. [CrossRef] [PubMed]

38. Konno, H.; Yamauchi, S.; Berglund, A.; Putney, R.M.; Mulé, J.J.; Barber, G.N. Suppression of STING signaling through epigenetic silencing and missense mutation impedes DNA damage mediated cytokine production. Oncogene 2018, 37, 2037-2051. [CrossRef]

39. Tsai, H.-J.; Choudhry, S.; Naqvi, M.; Rodriguez-Cintron, W.; Burchard, E.G.; Ziv, E. Comparison of three methods to estimate genetic ancestry and control for stratification in genetic association studies among admixed populations. Qual. Life Res. 2005, 118, 424-433. [CrossRef] [PubMed]

40. Tang, D.; Kryvenko, O.N.; Mitrache, N.; Do, K.C.; Jankowski, M.; Chitale, D.A.; Trudeau, S.; Rundle, A.; Belinsky, S.A.; Rybicki, B.A. Methylation of the RARB gene increases prostate cancer risk in black Americans. J. Urol. 2013, 190, 317-324. [CrossRef]

41. Pan, J.; Chen, J.; Zhang, B.; Chen, X.; Huang, B.; Zhuang, J.; Mo, C.; Qiu, S. Association between RASSF1A promoter methylation and prostate cancer: A systematic review and meta-analysis. PLoS ONE 2013, 8, e75283. 
42. Cheong, A.; Zhang, X.; Cheung, Y.-Y.; Tang, W.-Y.; Chen, J.; Ye, S.-H.; Medvedovic, M.; Leung, Y.K.; Prins, G.S.; Ho, S.-M. DNA methylome changes by estradiol benzoate and bisphenol A links early-life environmental exposures to prostate cancer risk. Epigenetics 2016, 11, 674-689. [CrossRef]

43. Kachroo, N.; Valencia, T.; Warren, A.Y.; Gnanapragasam, V.J. Evidence for downregulation of the negative regulator SPRED2 in clinical prostate cancer. Br. J. Cancer 2012, 108, 597-601. [CrossRef] [PubMed]

44. Portela, P.; Jobim, L.F.; Salim, P.H.; Koff, W.J.; Wilson, T.J.; Jobim, M.R.; Schwartsmann, G.; Roesler, R.; Jobim, M. Analysis of KIR gene frequencies and HLA class I genotypes in prostate cancer and control group. Int. J. Immunogenet. 2012, 39, 423-428. [CrossRef] [PubMed]

45. Sayaman, R.W.; Saad, M.; Thorsson, V.; Hu, D.; Hendrickx, W.; Roelands, J.; Porta-Pardo, E.; Mokrab, Y.; Farshidfar, F.; Kirchoff, T.; et al. Germline genetic contribution to the immune landscape of cancer. Immunity 2021, 54, 367.e8-386.e8. [CrossRef] [PubMed]

46. Sakabe, T.; Tsuchiya, H.; Kanki, K.; Azumi, J.; Gonda, K.; Mizuta, Y.; Yamada, D.; Wada, H.; Shomori, K.; Nagano, H.; et al Identification of the genes chemosensitizing hepatocellular carcinoma cells to interferon-alpha/5-fluorouracil and their clinical significance. PLoS ONE 2013, 8, e56197. [CrossRef]

47. Arai, T.; Miyoshi, Y.; Kim, S.J.; Taguchi, T.; Tamaki, Y.; Noguchi, S. Association of GSTP1 CpG islands hypermethylation with poor prognosis in human breast cancers. Breast Cancer Res. Treat. 2006, 100, 169-176. [CrossRef] [PubMed]

48. Pandey, M.; Shukla, S.; Gupta, S. Promoter demethylation and chromatin remodeling by green tea polyphenols leads to re-expression of GSTP1 in human prostate cancer cells. Int. J. Cancer 2010, 126, 2520-2533. [CrossRef] [PubMed]

49. Mian, O.Y.; Khattab, M.H.; Hedayati, M.; Coulter, J.; Abubaker-Sharif, B.; Schwaninger, J.M.; Veeraswamy, R.K.; Brooks, J.D.; Hopkins, L.; Shinohara, D.B.; et al. GSTP1 Loss results in accumulation of oxidative DNA base damage and promotes prostate cancer cell survival following exposure to protracted oxidative stress. Prostate 2015, 76, 199-206. [CrossRef] [PubMed]

50. Enokida, H.; Shiina, H.; Urakami, S.; Igawa, M.; Ogishima, T.; Pookot, D.; Li, L.-C.; Tabatabai, Z.L.; Kawahara, M.; Nakagawa, M.; et al. Ethnic group-related differences in CpG hypermethylation of the GSTP1 gene promoter among African-American, Caucasian and Asian patients with prostate cancer. Int. J. Cancer 2005, 116, 174-181. [CrossRef] [PubMed]

51. Cho, N.-Y.; Kim, B.-H.; Choi, M.; Yoo, E.J.; Moon, K.C.; Cho, Y.-M.; Kim, D.; Kang, G.H. Hypermethylation of CpG island loci and hypomethylation of LINE-1 and Alu repeats in prostate adenocarcinoma and their relationship to clinicopathological features. J. Pathol. 2006, 211, 269-277. [CrossRef] [PubMed]

52. Park, J.Y. Promoter hypermethylation as a biomarker in prostate adenocarcinoma. Method. Mol. Biol. 2015, 1238, $607-625$.

53. Kirby, M.K.; Ramaker, R.C.; Roberts, B.S.; Lasseigne, B.N.; Gunther, D.S.; Burwell, T.C.; Davis, N.S.; Gulzar, Z.G.; Absher, D.M.; Cooper, S.J.; et al. Genome-wide DNA methylation measurements in prostate tissues uncovers novel prostate cancer diagnostic biomarkers and transcription factor binding patterns. BMC Cancer 2017, 17, 273. [CrossRef] [PubMed]

54. Katoh, M.; Katoh, M. MGC29506 gene, frequently down-regulated in intestinal-type gastric cancer, encodes secreted-type protein with conserved cysteine residues. Int. J. Oncol. 2003, 23, 235-241. [CrossRef] [PubMed]

55. Xia, L.; Shen, C.; Fu, Y.; Tian, L.; Chen, M. MGC29506 induces cell cycle arrest and is downregulated in gastric cancer. Cell. Immunol. 2013, 281, 31-36. [CrossRef] [PubMed]

56. Wang, X.-Q.; Xu, S.-W.; Wang, W.; Piao, S.-Z.; Mao, X.L.; Zhou, X.-B.; Wang, Y.; Wu, W.-D.; Ye, L.-P.; Li, S.-W. Identification and validation of a novel DNA damage and DNA repair related genes-based signature for colon cancer prognosis. Front. Genet. 2021, 12, 635863. [CrossRef] [PubMed]

57. Alvi, M.A.; Liu, X.; O’Donovan, M.; Newton, R.; Wernisch, L.; Shannon, N.B.; Shariff, K.; di Pietro, M.; Bergman, J.J.G.H.M.; Ragunath , K.; et al. DNA methylation as an adjunct to histopathology to detect prevalent, inconspicuous dysplasia and early-stage neoplasia in Barrett's esophagus. Clin. Cancer Res. 2013, 19, 878-888. [CrossRef] [PubMed]

58. Chandrashekar, D.S.; Bashel, B.; Balasubramanya, S.A.H.; Creighton, C.J.; Ponce-Rodriguez, I.; Chakravarthi, B.V.S.K.; Varambally, S. A portal for facilitating tumor subgroup gene expression and survival analyses. Neoplasia 2017, 19, 649-658. [CrossRef]

59. Carastro, L.M.; Lin, H.-Y.; Park, H.Y.; Kim, D.; Radlein, S.; Hampton, K.K.; Hakam, A.; Zachariah, B.; Pow-Sang, J.; Park, J.Y Role of p73 dinucleotide polymorphism in prostate cancer and p73 protein isoform balance. Prostate Cancer 2014, $2014,129582$. [CrossRef] [PubMed]

60. Martin, M.P.; Borecki, I.B.; Zhang, Z.; Nguyen, L.; Ma, D.; Gao, X.; Qi, Y.; Carrington, M.; Rader, J.S. HLA-Cw group 1 ligands for KIR increase susceptibility to invasive cervical cancer. Immunogenetics 2010, 62, 761-765. [CrossRef]

61. Lee, C.-W.; Wong, L.L.-Y.; Tse, E.Y.-T.; Liu, H.F.; Leong, V.Y.-L.; Lee, J.M.-F.; Hardie, D.G.; Ng, I.O.-L.; Ching, Y.-P. AMPK promotes p53 acetylation via phosphorylation and inactivation of SIRT1 in liver cancer cells. Cancer Res. 2012, 72, 4394-4404. [CrossRef] [PubMed]

62. William, W.N.; Kim, J.-S.; Liu, D.D.; Solis, L.; Behrens, C.; Lee, J.J.; Lippman, S.M.; Kim, E.S.; Hong, W.K.; Wistuba, I.I.; et al. The impact of phosphorylated AMP-activated protein kinase expression on lung cancer survival. Ann. Oncol. 2012, 23, 78-85. [CrossRef] 